\title{
Analysis of Cell-free Circulating DNA Fragment Size and Level in Patients With Lumbar Degenerative Disease
}

\author{
Akihiko Hiyama ( $\nabla$ a.hiyama@tokai-u.jp ) \\ Tokai University School of Medicine \\ Daisuke Sakai \\ Tokai University School of Medicine
}

Satoshi Nomura

Tokai University School of Medicine

Hiroyuki Katoh

Tokai University School of Medicine

Masahiko Watanabe

Tokai University School of Medicine

\section{Research Article}

Keywords: Cell-free circulating DNA, low back pain, lumbar degenerative disease, numeric rating scale, lateral lumbar interbody fusion

Posted Date: May 12th, 2021

DOI: https://doi.org/10.21203/rs.3.rs-497374/v1

License: (c) (i) This work is licensed under a Creative Commons Attribution 4.0 International License. Read Full License 


\section{Abstract}

Background: Cell-free circulating DNA (cfDNA), which can be extracted by liquid biopsy, has been studied as a noninvasive biomarker for various diseases. The potential of cfDNA fragment size and level as a marker for low back pain (LBP) has never been studied. We investigated whether cfDNA is a biomarker of LBP severity in patients with a lumbar degenerative disease (LDD).

Methods: Blood samples were obtained from patients with LDD $(n=21)$ before and immediately after spinal surgery. Plasma DNA was isolated and examined for cfDNA fragment size and concentration. A cohort of healthy volunteers $(n=5)$ constituted the control group.

Results: The cfDNA fragment size tended to be shorter in patients than in healthy controls, but this difference was not significant $(P=.224)$. cfDNA level was significantly higher in LDD patients (mean $0.642 \pm 0.199 \mathrm{ng} / \mathrm{mL}$, range $0.302-1.150 \mathrm{ng} / \mathrm{mL}$ ) than in healthy controls (mean $0.429 \pm 0.064 \mathrm{ng} / \mathrm{mL}$, range $0.366-0.506 \mathrm{ng} / \mathrm{mL})(P=.029)$. cfDNA level correlated positively with present pain $(\mathrm{r}=.421, P=$ .036), maximum pain $(\mathrm{r}=.419, P=.037)$, average pain $(\mathrm{r}=.566, P=.003)$, low back pain $(\mathrm{r}=.403, P=$ $.041)$, leg pain $(r=.480, P=.013)$, and leg numbness $(r=.455, P=.020)$. cfDNA fragment size did not differ from before to after surgery, but cfDNA level increased postoperatively in patients with LDD.

Conclusions: This was the first study investigating whether cfDNA fragment size and level are associated with pain, including LBP, in patients with LDD. Our findings suggest that cfDNA level may be an objective indicator of pain and surgical invasiveness in patients with LDD.

\section{Background}

Low back pain (LBP) is among the ten most frequent reasons for visiting a primary care practitioner across all adult age groups [1]. In industrialized countries, the lifetime prevalence of LBP is $70-90 \%$, and the 1-year prevalence is 15-45\% [2]. Clinically, LBP is caused by multiple triggers, but the most common etiologies of LBP include intervertebral disc herniation, lumbar spinal stenosis, and lumbar degenerative disease (LDD). LBP management comprises various intervention strategies, including surgery and nonmedical interventions such as exercise, behavioral therapy, and alternative therapies.

Pharmacological intervention is the most frequently recommended intervention for LBP [3]. In addition to nonsteroidal anti-inflammatory drugs and muscle relaxants, opioids, tramadol, benzodiazepines, and gabapentin (for radiculopathy) have now been added possible pharmacological interventions. Spine surgeons are prescribing drugs that are likely to be useful for symptomatic treatment while considering the disease's pathophysiology that causes LBP.

The severity of LBP is evaluated according to the therapeutic effects of treatment, whose effectiveness is based on subjective evaluation and, at present, there are no biomarkers for evaluating LBP objectively. Various evaluation scales, such as the visual analog scale and numeric rating scale (NRS), are used to evaluate LBP. Basic research using animal models is limited by the inability to identify pain in the animals. Objective evaluation with a measuring device or biomarker that can measure pain is required; 
however, the lack of a biomarker to quantify pain is a barrier to LBP research. Cell-free circulating DNA (cfDNA) is generally found in small amounts in the blood of healthy people. Elevated cfDNA levels have been reported in patients with malignant or autoimmune diseases, myocardial infarction, and trauma [46] and suggested that the release of cfDNA into the circulation may be useful, although nonspecific, a marker of tissue injury. The use of cfDNA level as a potential marker of the severity of LBP has not been studied. Therefore, the purpose of this study was to investigate whether cfDNA level is a biomarker of LBP severity in patients with LDD.

\section{Material And Methods Participants}

The inclusion criteria included patients 20 years of age or older and the need for surgery in the lumbar spine (L1-L2 to L5-S1) to treat LDD. All patients were diagnosed based on a detailed history, neurological and radiographic examinations, myelogram results, computed tomography (CT) scans after myelography, and/or magnetic resonance imaging (MRI).

In brief, participants were eligible if they had undergone spinal surgery, been diagnosed with a degenerative spinal disease based on physical and imaging findings, had received sufficient explanation about participating in this study, and had voluntarily provided written consent.

The exclusion criteria included severe mental illness, difficulty standing or moving because of severe impairment of paralysis, prior (within five years) or current cancer diagnosis, suspect a current infection, or the presence of a condition considered by the principal investigator to be a contraindication for inclusion in this study.

The authors provided information to the patients about the use of samples obtained during surgery, and patients were asked to read and sign the consent forms before surgery. Demographic data and clinical data were obtained after inclusion in the study.

Between October 2020 and February 2021, blood was collected from 21 patients (13 males, 8 females; age, $72.2 \pm 8.2$ years) diagnosed with LDD accompanied by LBP, leg pain (LP), and/or leg numbness (LN) and hospitalized for surgical purposes. Blood samples were collected from 5 healthy volunteers (4 males, 1 female; age, $38.0 \pm 7.8$ years) who had never experienced LBP and were selected as the control group. Table 1 summarizes surgical records for LDD patients and the CRP data on the first day after surgery.

\section{Procedures}

A $10 \mathrm{~mL}$ blood sample was collected in Streck Cell-Free DNA BCT $®$ (Streck, La Vista, NE, USA). In the LDD patients, blood samples were collected immediately after induction of general anesthesia (baseline) and immediately after surgery. 
Whole blood was separated from Streck Cell-Free DNA BCT ${ }^{\circledR}$ by centrifugation at 1,700 $\mathrm{g}$ for $10 \mathrm{~min}$ at $25^{\circ} \mathrm{C}$. The upper plasma layer was removed, transferred to a new conical tube, and centrifuged at $5000 \mathrm{~g}$ for 10 min. Plasma samples were stored at $-80^{\circ} \mathrm{C}$ until use. C-reactive protein (CRP) concentration was measured in postoperative blood samples as an indicator of the invasiveness of the surgery.

\section{Determination of cfDNA fragment size and level}

The method uses only $1 \mu$ l of DNA from plasma for one test, is very sensitive, with a dynamic range of 0$400 \mathrm{ng} / \mu$ l human genomic DNA, and yields highly reproducible results. Qubit 3.0, with a dsDNA assay kit, was used to measure the total amount of genomic DNA.

cfDNA fragment size was determined for each sample with an Agilent 2100 Bioanalyzer and the Agilent High Sensitivity DNA chip, according to the manufacturer's instructions. The fragment size of cfDNA was determined with the Agilent 2100 Bioanalyzer software and defined as the main peak mode (corresponding to one nucleosome plus linker, derived from apoptotic cells) electropherogram. cfDNA level was performed using the Qubit fluorometer 3.0 (Invitrogen, Life Technologies) combined with the Qubit dsDNA HS Assay Kit (Invitrogen, Life technologies; cat \#Q32851, lot \#1724782). As per the manufacturer's instructions, a standard curve was prepared using the 0 and $10 \mathrm{ng} / \mu \mathrm{L}$ Qubit standards provided in the kit. For all cfDNA extractions, $1 \mu \mathrm{L}$ of the sample was diluted in $199 \mu \mathrm{L}$ Qubit working solution before measurement.

\section{Pain intensity}

The intensity of pain was assessed using a 3-level NRS [7]. Each patient was asked to grade the actual pain level experienced at present (present pain; $N_{R} S_{\text {Present }}$ ), maximum pain level experienced in the past four weeks (maximum pain; $N_{R S} S_{\text {Max }}$ ), and average pain level experienced in the past four weeks (average pain; $\mathrm{NRS}_{\text {Ave }}$ ) on a scale of 0 to 10 (where 0 is no pain and 10 is the worst pain imaginable). NRS scores were obtained for LBP $\left(\mathrm{NRS}_{\mathrm{LBP}}\right), \mathrm{LP}\left(\mathrm{NRS}_{\mathrm{LP}}\right)$, and LN $\left(\mathrm{NRS}_{\mathrm{LN}}\right)$. Spearman's correlational analysis assessed the relationships between the patients' cfDNA levels and their respective NRS scores.

\section{Statistical analysis}

Statistical analyses were performed using IBM SPSS Statistics (version 23.0; IBM Corp., Armonk, NY, USA). All values are expressed as mean \pm standard deviation. The Shapiro-Wilk test was used to confirm the normality of the data distribution. For the primary analysis, Student's t-test or the Mann-Whitney $U$ test was used to compare the two groups. The Student's t-test was used to analyze normally distributed data and the Mann-Whitney $U$ test for nonnormally distributed data. The Mann-Whitney $U$ test was used to compare cfDNA fragment size, and Student's t-test was used to compare cfDNA levels between control and patient samples. Pearson's product-moment correlation analysis or Spearman's product-moment correlational analysis was used to identifying significant associations.

We used the G-Power Analysis software program to determine the power for sample size validity (G*Power 3.1). Post-hoc analysis using G*Power 3.1 was performed to detect the correlation of subjects 
and the difference between two independent groups.

The type 1 error was set at $5 \%$ for all statistical analyses, and $\mathrm{P}<.05$ was significant.

\section{Results}

The baseline characteristics and clinical data of the patient and healthy control groups are summarized in Table 2. The pain intensity scores for the 21 patients were $6.8 \pm 2.2$ for actual pain level experienced at present (present pain; NRS Present), $8.0 \pm 2.3$ for maximum pain level experienced in the past four weeks (maximum pain; $\mathrm{NRS}_{\mathrm{Max}}$ ), and $7.0 \pm 2.3$ for average pain level experienced in the past four weeks (average pain; $\mathrm{NRS}_{\text {Ave }}$ ). All patients reported LBP (mean NRS ${ }_{\mathrm{LBP}} 6.5 \pm 2.6$ ), LP (mean NRS $\mathrm{LP}_{\mathrm{LP}} 6.8 \pm 2.8$ ), and $L N$ (mean NRS $S_{L N} 6.8 \pm 2.4$ ). The mean preoperative CRP was $0.30 \mathrm{mg} / \mathrm{L}$ (range $0.09-1.76 \mathrm{mg} / \mathrm{L}$ ) (data not shown).

The operative levels were as follows: L2 -3 for five patients, L3-4 for eight patients, L4-5 for sixteen patients, and L5-S1 for one patient. The mean operative time was 119.0 $\pm 47.9 \mathrm{~min}$ (range 64-293 min). The mean estimated blood loss was $125.0 \pm 151.1 \mathrm{~mL}$ (range 2-695 mL). The mean length of hospital stay was $17.3 \pm 4.5$ days (range 10-27 days). The mean postoperative CRP concentration was $2.82 \mathrm{mg} / \mathrm{L}$ (range $0.37-5.04 \mathrm{mg} / \mathrm{L}$ ).

\section{Measurements of cfDNA Fragment Size and cfDNA Level}

cfDNA fragment size and cfDNA levels were measured in plasma samples from patients and healthy controls (Fig. 1 and Fig. 2). The cfDNA fragment size tended to be shorter in patients (mean $166.0 \pm 13.1$ bp, range 135-180 bp) than in healthy controls (mean 172.6 $\pm 4.0 \mathrm{bp}$, range 166-176 bp), but this difference was not significant $(P=.224)$. Plasma cfDNA level was significantly higher in LDD patients (mean $0.642 \pm 0.199 \mathrm{ng} / \mu \mathrm{L}$, range $0.302-1.150 \mathrm{ng} / \mu \mathrm{L}$ ) than in healthy controls (mean $0.429 \pm 0.064 \mathrm{ng} /$ $\mu \mathrm{L}$, range $0.366-0.506 \mathrm{ng} / \mu \mathrm{L})(P=.029$, effect size $\mathrm{d}=1.578$, Power $(1-\beta$ error prob $)=.860)$.

\section{Changes in cfDNA Fragment Size and cfDNA Levels from before to after Surgery}

cfDNA fragment size and cfDNA levels were measured in plasma samples obtained from patients before and after surgery. The median cfDNA fragment did not change from before (mean $166.0 \pm 13.1 \mathrm{bp}$, range $135-180 \mathrm{bp}$ ) to after surgery (mean $168.4 \pm 11.5 \mathrm{bp}$, range 144-182 bp) $(P=.110)$. By contrast, cfDNA level increased significantly from before (mean $0.642 \pm 0.199 \mathrm{ng} / \mu \mathrm{L}$, range $0.302-1.150 \mathrm{ng} / \mu \mathrm{L}$ ) to after surgery (mean $7.110 \pm 4.969 \mathrm{ng} / \mu \mathrm{L}$, range $0.72-23.8 \mathrm{ng} / \mu \mathrm{L})(P<.001$, effect size $d=1.356$, Power $(1-\beta$ error prob) $=.999)($ Fig. 3).

\section{Correlational analysis between cfDNA levels and related factors}


A power analysis performed to detect the correlation (effect size $d=0.5$, alpha $=0.05$, total sample size $=$ 26 , two-tailed) showed 0.806 . We firstly performed correlational analysis between age and cfDNA levels but found no significant correlation between age and cfDNA levels ( $r=.325, P=.106$, data not shown). Table 3 shows the correlations between cfDNA level or cfDNA fragment size and various indictors of pain and between postoperative cfDNA level and related factors. cfDNA level $(n=26)$ correlated significantly with NRS Present $(r=.421, P=.036), \mathrm{NRS}_{\mathrm{Max}}(\mathrm{r}=.419, P=.037), \mathrm{NRS}_{\text {Ave }}(\mathrm{r}=.566, P=.003), \mathrm{NRS}_{\text {LBP }}(\mathrm{r}=.403$, $P=.041), \mathrm{NRS}_{\mathrm{LP}}(\mathrm{r}=.480, P=.013)$, and $\mathrm{NRS}_{\mathrm{LN}}(\mathrm{r}=.455, P=.020)$. Figure 4 demonstrate the distribution of each NRS and cfDNA level. cfDNA fragment size did not correlate significantly with any of these indicators of pain.

Postoperative cfDNA level did not correlate significantly with other factors such as operative time ( $r$ $=.312, P=.168)$, blood loss $(r=.364, P=.105)$, length of hospital stay $(r=.203, P=.405)$, postoperative CRP concentration $(r=.371, P=.097)$, age $(r=-.113, P=.625)$, and gender $(r=.0016, P=.944)$.

\section{Discussion}

This is the first study investigating whether cfDNA fragment size and level in LDD patients are associated with various aspects of pain, such as LBP. Elevated cfDNA levels have been reported to be associated with a variety of pathological processes [8-10]. For example, an association between cfDNA and pain has been reported in patients with sickle-cell disease [11]. That study reported that cfDNA levels in patients' plasma were higher during acute painful episodes with steady-state levels.

Against this background, we speculated that the cfDNA fragment size and levels in LDD patients might also vary depending on the degree of pain. We first compared cfDNA fragment size and levels in LDD patients and healthy controls. cfDNA fragment size tended to be shorter in patients than in controls, but this difference was not significant. Previous reports on cfDNA in cancer patients showed that cfDNA size is shorter in fragments originating in tumor cells than in those from nonmalignant cells [12-14]. An increased number of short cfDNA fragments may reflect an increase in tumor DNA level relative to nontumor DNA in the blood of cancer patients. It is thought that DNA methylation may affect cfDNA size $[15,16]$. However, the mechanism responsible for the shorter cfDNA fragment size is not well understood.

We also found that cfDNA level was higher in patients with LDD than in healthy controls. Interestingly, the cfDNA level correlated positively with some NRS scores. It is difficult to treat LN in patients with LDD, and postoperative symptoms may remain, and the lack of an index exacerbates this problem to evaluate pain objectively. Our results suggest that the cfDNA level may help in quantifying LBP and LP, and LN.

Subsequently, we investigated whether spinal surgery's invasiveness affects cfDNA fragment size and level by measuring these before and after surgery in LDD patients. We found no significant change in cfDNA fragment size from before to after surgery, although cfDNA level increased postoperatively in all patients. A previous study reported that lactate dehydrogenase (LDH), an enzyme found in various living cells, functions as a biomarker of injury and disease [17]. That study found that elevated cfDNA levels 
and elevated LDH levels were associated with tissue damage, directly associated with pain [18]. Patients were receiving chemotherapy exhibit significant increases in cfDNA levels $24 \mathrm{~h}$ and eight days after chemotherapy [19] and several cycles after chemotherapy [20]. These data led us to investigate whether the invasiveness of spinal surgery would be related to an increase in cfDNA level. In recent years, minimally invasive spinal surgery has attracted much attention. However, surgical time, bleeding loss, length of hospital stay, and postoperative CRP levels are often used to evaluate surgical invasiveness [21]. Surgical procedures induce a complex stress response proportional to the magnitude of the injury, operating time, and intraoperative blood loss. The adverse metabolic and hemodynamic effects of this stress response can cause many problems during the perioperative period. Thus, decreasing the stress response to surgery is a key factor for improving clinical outcomes.

Circulating cfDNA in the blood may be helpful for objectively assessing the body's response to an invasive procedure such as surgery, and its measurement may have potential value for diagnosis and prognosis. Our results suggest that cfDNA level may be a new biomarker for assessing the invasiveness in spinal surgery. A previous report noted that the extent to which postoperative CRP level is elevated seems to depend upon the severity of the procedure [22]. However, there was no correlation between cfDNA level and postoperative CRP level. Further research is needed to clarify whether cfDNA level is related to CRP level and postoperative pain.

A limitation of this study is the small sample size in both the patient and control groups. However, the post hoc power analysis at the cfDNA level was 0.8 or higher between LDD patients and the healthy controls. In addition, the ages of the two groups differed significantly. However, it is difficult to obtain samples from patients with an average age of 70 who do not experience pain because many older people have some form of locomotor disorder. Another limitation is that we did not compare cfDNA fragment size and levels for different surgical procedures. To evaluate surgical invasiveness more concretely, we think it is necessary to compare cfDNA size and level between decompression surgery and fusion surgery or traditional open surgery and minimally invasive spinal surgery. Additionally, comorbidities can affect the cfDNA fragment size and level; to reduce the possible bias. It would help control for comorbidities in future studies. Finally, we have not investigated the role of cfDNA in predicting symptom response to treatment and sustainability in the long term.

\section{Conclusions}

In summary, cfDNA level was higher in patients with LDD exhibit than in healthy controls. cfDNA levels increased significantly after spinal surgery and correlated positively with various pains, including leg pain or numbness. However, the clinical relevance of an elevated cfDNA level after spinal surgery is unknown, and the utility of cfDNA as a marker of mechanical tissue injury remains unproven. Further studies involving serial measurements in more significant, independent patient populations are needed to determine whether cfDNA quantification is appropriate for clinical use.

\section{Abbreviations}


cfDNA, Cell-free circulating DNA; LBP, low back pain; LDD, lumbar degenerative disc disease; CT, computed tomography; MRI, magnetic resonance imaging; LP, leg pain; LN, leg numbness; CRP, C-reactive protein

\section{Declarations}

\section{Ethics approval and consent to participate}

The Committee approved the study on Ethics and the Institutional Review Board of Tokai University School of Medicine, the House Clinical Study Committee, and the Profit Reciprocity Committee (20R-263). The study was conducted following the principles outlined in the Helsinki declaration [23].

All the participants provided informed written consent for the provision of blood sampling and clinical data.

\section{Consent for publication}

Not applicable.

\section{Availability of data and materials}

Data were available upon request from the corresponding author.

\section{Competing interests}

The authors $\mathrm{AH}, \mathrm{DS}, \mathrm{SN}, \mathrm{HK}$, and MW declare that they have no competing interests.

\section{Funding}

This study was supported in part by the Research and Study Program of the Tokai University Educational System General Research Organization and by a Grant-in-Aid for Scientific Research (KAKENHI).

\section{Authors' contributions}

All authors have read and approved the manuscript. AH conceived and designed the study and interpreted the results; DS acquired the data and interpreted the results; SN analyzed the data and interpreted the results; HK acquired the data and interpreted the results; MW conceived and designed the study and interpreted the results.

\section{Acknowledgments}

We much appreciate the helpful advice of Dr. Masayuki Tanaka and the technical assistance of Dr. Kanae Kitatani and Dr. Tadayuki Sato.

\section{References}


1. Blount BW, Hart G, Ehreth JL: A description of the content of army family practice. J Am Board Fam Pract 1993, 6(2):143-152.

2. Papageorgiou AC, Croft PR, Ferry S, Jayson MI, Silman AJ: Estimating the prevalence of low back pain in the general population. Evidence from the South Manchester Back Pain Survey. Spine (Phila Pa 1976) 1995, 20(17):1889-1894.

3. Vogt MT, Kwoh CK, Cope DK, Osial TA, Culyba M, Starz TW: Analgesic usage for low back pain: impact on health care costs and service use. Spine (Phila Pa 1976) 2005, 30(9):1075-1081.

4. Fu X, Wan S, Hann HW, Myers RE, Hann RS, Au J, Chen B, Xing J, Yang H: Relative telomere length: a novel non-invasive biomarker for the risk of non-cirrhotic hepatocellular carcinoma in patients with chronic hepatitis B infection. Eur J Cancer 2012, 48(7):1014-1022.

5. Jylhävä J, Lyytikäinen LP, Kähönen M, Hutri-Kähönen N, Kettunen J, Viikari J, Raitakari OT, Lehtimäki T, Hurme M: A genome-wide association study identifies UGT1A1 as a regulator of serum cell-free DNA in young adults: The Cardiovascular Risk in Young Finns Study. PLoS One 2012, 7(4):e35426.

6. Lögters T, Paunel-Görgülü A, Zilkens C, Altrichter J, Scholz M, Thelen S, Krauspe R, Margraf S, Jeri T, Windolf $\mathrm{J}$ et al: Diagnostic accuracy of neutrophil-derived circulating free DNA (cf-DNA/NETs) for septic arthritis. J Orthop Res 2009, 27(11):1401-1407.

7. Dworkin RH, Turk DC, Farrar JT, Haythornthwaite JA, Jensen MP, Katz NP, Kerns RD, Stucki G, Allen RR, Bellamy $\mathrm{N}$ et al: Core outcome measures for chronic pain clinical trials: IMMPACT recommendations. Pain 2005, 113(1-2):9-19.

8. Clementi A, Virzì GM, Brocca A, Pastori S, de Cal M, Marcante S, Granata A, Ronco C: The Role of CellFree Plasma DNA in Critically III Patients with Sepsis. Blood Purif 2016, 41(1-3):34-40.

9. Gögenur M, Burcharth J, Gögenur I: The role of total cell-free DNA in predicting outcomes among trauma patients in the intensive care unit: a systematic review. Crit Care 2017, 21(1):14.

10. Schwarzenbach H, Hoon DS, Pantel K: Cell-free nucleic acids as biomarkers in cancer patients. Nat Rev Cancer 2011, 11(6):426-437.

11. Al-Humood S, Zueriq R, Al-Faris L, Marouf R, Al-Mulla F: Circulating cell-free DNA in sickle cell disease: is it a potentially useful biomarker? Arch Pathol Lab Med 2014, 138(5):678-683.

12. DiehI F, Li M, Dressman D, He Y, Shen D, Szabo S, Diaz LA, Jr., Goodman SN, David KA, Juhl H et al: Detection and quantification of mutations in the plasma of patients with colorectal tumors. Proc Natl Acad Sci U S A 2005, 102(45):16368-16373.

13. Jiang P, Chan CW, Chan KC, Cheng SH, Wong J, Wong VW, Wong GL, Chan SL, Mok TS, Chan HL et al: Lengthening and shortening of plasma DNA in hepatocellular carcinoma patients. Proc Natl Acad Sci U S A 2015, 112(11):E1317-1325.

14. Mouliere F, Robert B, Arnau Peyrotte E, Del Rio M, Ychou M, Molina F, Gongora C, Thierry AR: High fragmentation characterizes tumour-derived circulating DNA. PLoS One 2011, 6(9):e23418.

15. Lapin M, Oltedal S, Tjensvoll K, Buhl T, Smaaland R, Garresori H, Javle M, Glenjen NI, Abelseth BK, Gilje $B$ et al: Fragment size and level of cell-free DNA provide prognostic information in patients with advanced pancreatic cancer. J Trans/ Med 2018, 16(1):300. 
16. Sun K, Jiang P, Wong AIC, Cheng YKY, Cheng SH, Zhang H, Chan KCA, Leung TY, Chiu RWK, Lo YMD: Size-tagged preferred ends in maternal plasma DNA shed light on the production mechanism and show utility in noninvasive prenatal testing. Proc Natl Acad Sci U S A 2018, 115(22):E5106-e5114.

17. Lahoud RM, O'Shea A, El-Mouhayyar C, Atre ID, Eurboonyanun K, Harisinghani M: Tumour markers and their utility in imaging of abdominal and pelvic malignancies. Clin Radiol 2021, 76(2):99-107.

18. de Souza Barbosa Ê, Santos Ibiapina HN, Rocha da Silva S, Costa AG, Val FF, Mendonça-da-Silva I, Carlos de Lima Ferreira L, Sartim MA, Monteiro WM, Cardoso de Melo G et al: Association of cfDNA levels and bothrops envenomation. Toxicon 2021, 192:66-73.

19. Swystun LL, Mukherjee S, Liaw PC: Breast cancer chemotherapy induces the release of cell-free DNA, a novel procoagulant stimulus. J Thromb Haemost 2011, 9(11):2313-2321.

20. Kwee S, Song MA, Cheng I, Loo L, Tiirikainen M: Measurement of circulating cell-free DNA in relation to 18F-fluorocholine PET/CT imaging in chemotherapy-treated advanced prostate cancer. Clin Trans/ Sci 2012, 5(1):65-70.

21. Houten JK, Tandon A: Comparison of postoperative values for C-reactive protein in minimally invasive and open lumbar spinal fusion surgery. Surg Neurol Int 2011, 2:94.

22. Al-Jabi Y, El-Shawarby A: Value of C-reactive protein after neurosurgery: a prospective study. $\mathrm{Br} \mathrm{J}$ Neurosurg 2010, 24(6):653-659.

23. World Medical Association Declaration of Helsinki: ethical principles for medical research involving human subjects. Jama 2013, 310(20):2191-2194.

\section{Tables}

Due to technical limitations, table 1-3 is only available as a download in the Supplemental Files section.

\section{Figures}

(A)

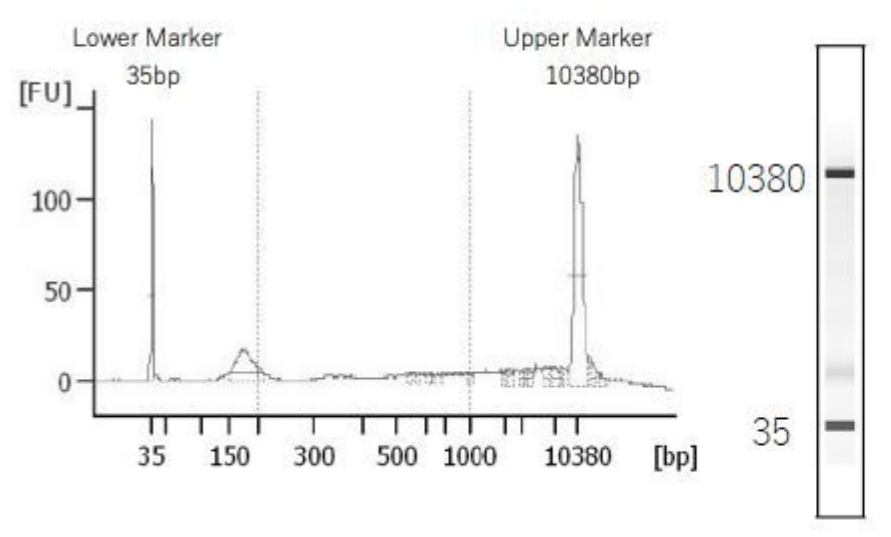

(B)

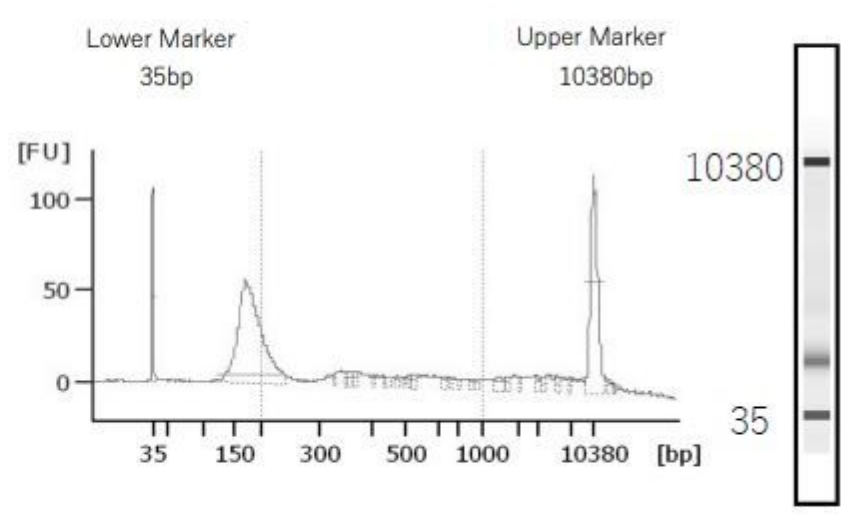


Figure 1

Distribution of cfDNA fragment size and cfDNA level in plasma. cfDNA from healthy controls $(A)$ and LDD patients (B) displayed larger fragment size (x-axis) and cfDNA level (y-axis). 35 bp and 10,380 bp are markers. LDD, lumbar degenerative disease.

(A)

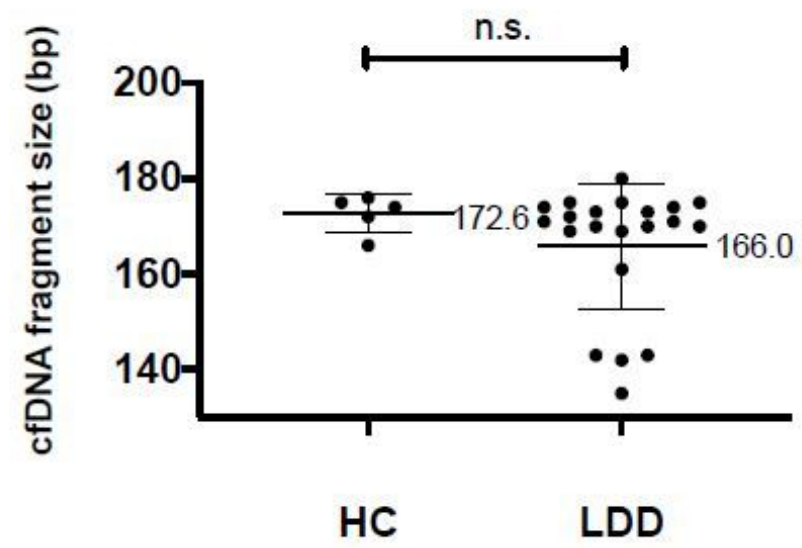

(B)

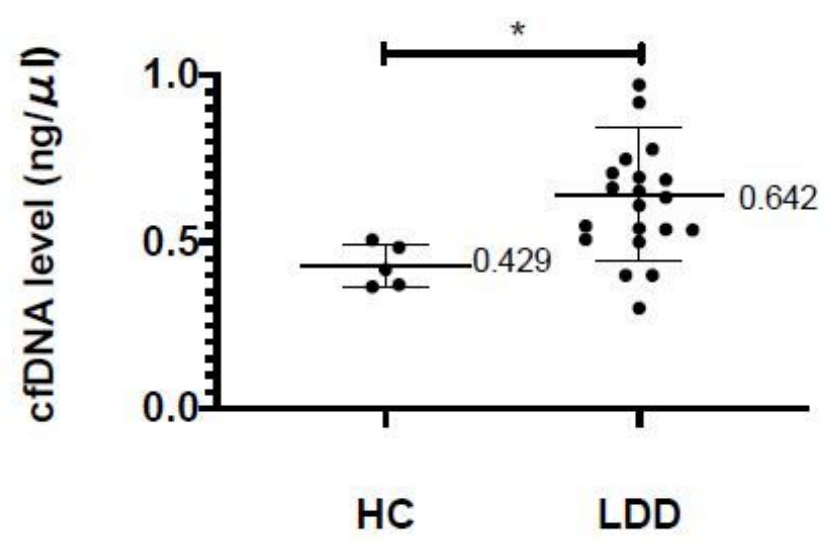

Figure 2

Distribution of cfDNA fragment size and cfDNA level in LDD patient and control samples. Each point shows differences in (A) cfDNA fragment size and (B) cfDNA level between samples from healthy controls $(H C ; n=5)$ and patients with a lumbar degenerative disease (LDD; $n=21)$. HC; healthy controls, LDD; lumbar degenerative disease n.s., not significant; $* P<0.05$.

(A)

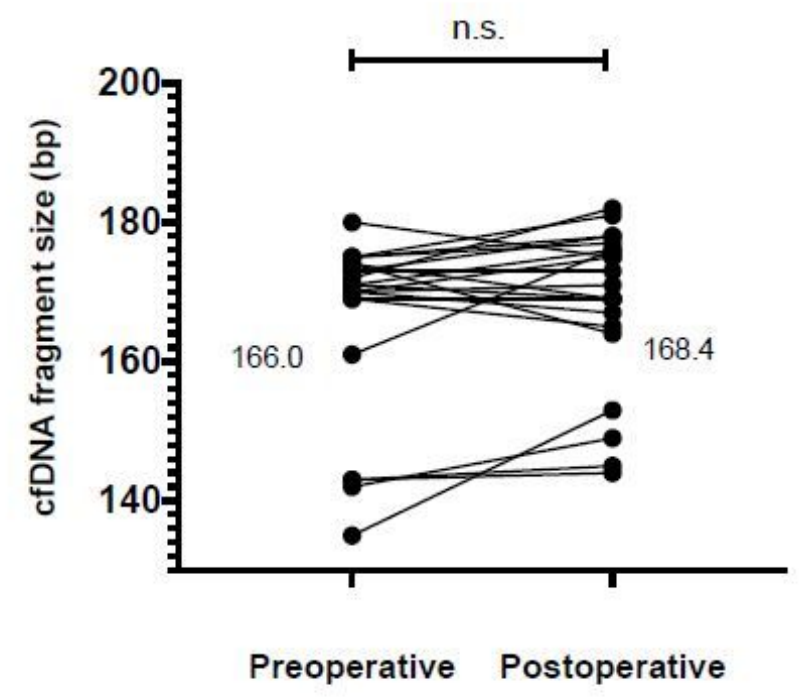

(B)

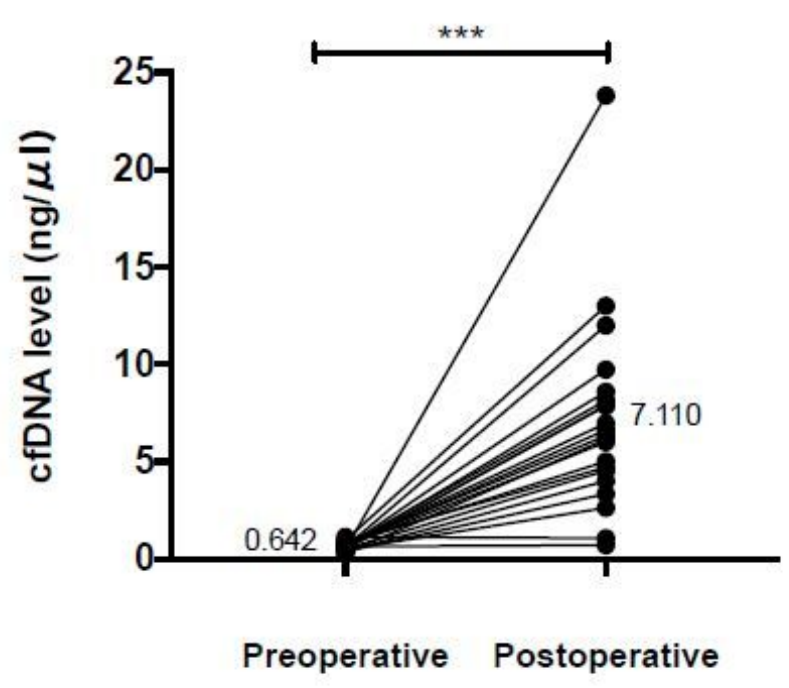




\section{Figure 3}

Changes in cfDNA fragment size and cfDNA levels from before to after surgery. Each point indicates (A) cfDNA fragment size and (B) cfDNA level before and after surgery for patients with an LDD $(n=21)$. The data are expressed as mean values. LDD, lumbar degenerative disease n.s., not significant; ${ }^{\star \star \star} P<0.001$.
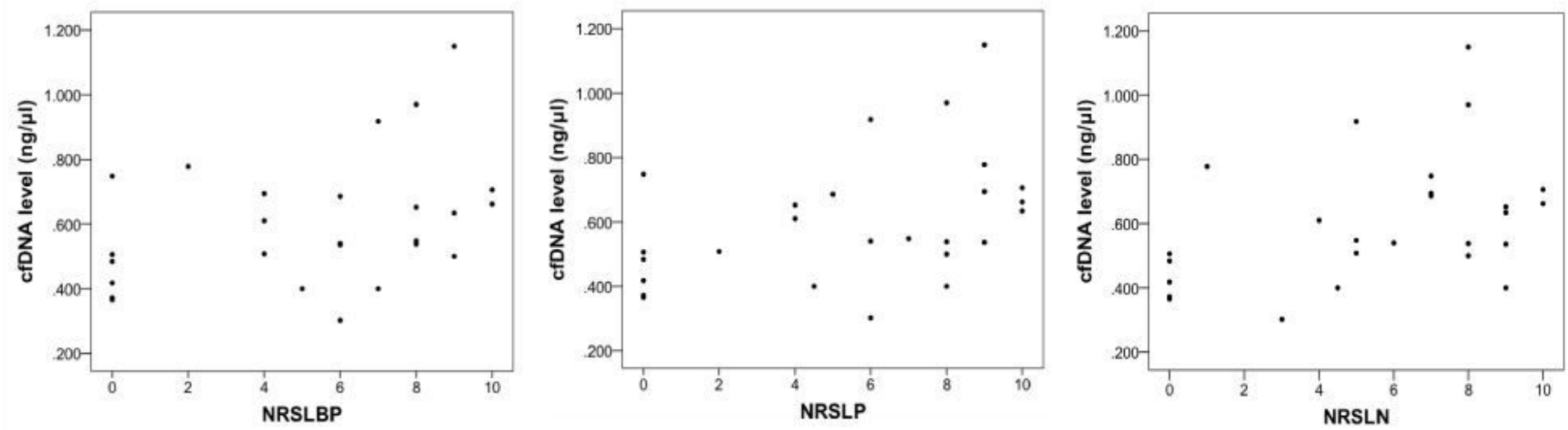

Figure 4

Scatter plot and Spearman's correlation between each pain and cfDNA level. Each point on the scatter plot represents one patient. NRS; Numeric Rating Scale, NRSLBP; NRS of low back pain, NRSLP; NRS of leg pain, NRSLN; NRS of leg numbness

\section{Supplementary Files}

This is a list of supplementary files associated with this preprint. Click to download.

- Table2.pdf

- Table3.pdf

- Table1.pdf 